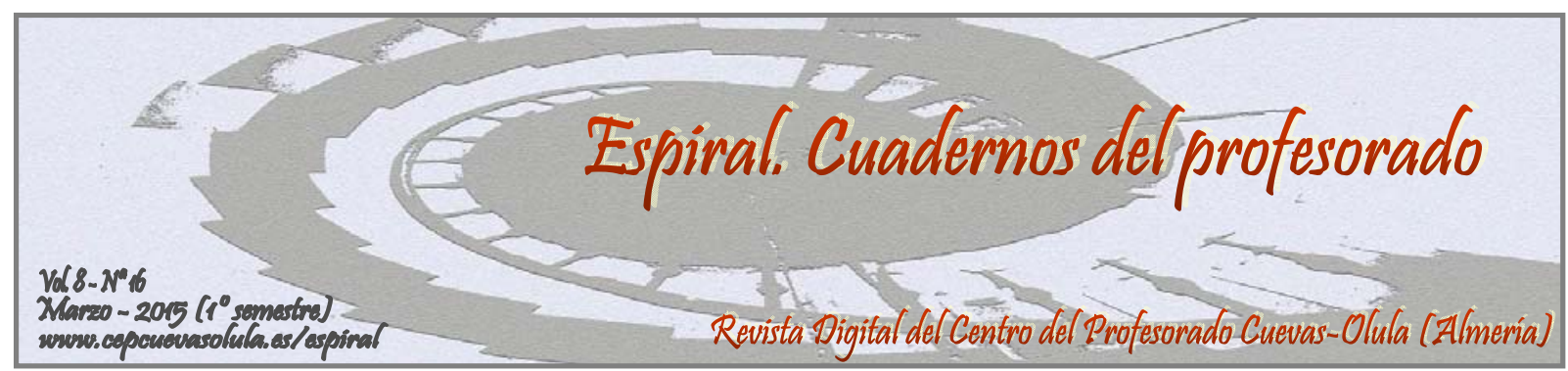

\title{
ESTUDIO EXPLORATORIO SOBRE DIFICULTADES EN EL APRENDIZAJE DE UNA SEGUNDA LENGUA: LA OPINIÓN DEL PROFESORADO
}

\section{EXPLORATORY STUDY ABOUT LEARNING DISABILITIES IN A FOREIGN LANGUAGE: TEACHERS' OPINION}

\section{Natalia Valero Molina y Gracia Jiménez-Fernández}

\author{
Departamento de Psicología Evolutiva y de la Educación, Facultad de Ciencias de la Educación, \\ Universidad de Granada, España
}

RESUMEN: Este artículo tiene como objetivo examinar la posible existencia de una dificultad específica para adquirir una segunda lengua (inglés). Para ello, se elaboró y aplicó una entrevista a docentes especialistas y tutores con el propósito de identificar a los alumnos con bajo rendimiento en inglés y analizar si dicho rendimiento se circunscribía solo al ámbito del aprendizaje de la segunda lengua o era generalizado a más áreas curriculares. Los resultados mostraron que, si bien el $79 \%$ de los escolares que no superan dicha materia presentan problemas generalizados, existe un 21\% que presentan una dificultad específica de adquisición del idioma extranjero. Se discuten las implicaciones educativas de la existencia de este tipo de problemática para el aprendizaje del inglés.

Palabras clave: dificultades de aprendizaje, adquisición de una segunda lengua, maestro especialista lengua extranjera, educación primaria.

ABSTRACT: The main aim of this paper is to examine the possible existence of a specific difficulty to learn a second language (English). Therefore, we developed and applied an interview for specialist teachers and tutors in order to identify students with low performance in English and analyse the causes of this failure. The results show that $79 \%$ of students who fail in English have widespread problems but there is a $21 \%$ with a specific learning disability in a second language. Educational implications of this specific problem for learning English are discussed.

Key words: learning disabilities, foreign language acquisition, foreign language teacher, Primary Education.

Valero Molina, N. y Jiménez-Fernández, G. (2015). Estudio exploratorio sobre dificultades en el aprendizaje de una segunda lengua: la opinión del profesorado. Espiral. Cuadernos del Profesorado, 8(16), 3-12. Disponible en: http://www.cepcuevasolula.es/espiral.

Fecha de recepción: 24/02/2015

Fecha de aceptación: 15/03/2015
Enviar correspondencia a: gracijf@ugr.es

Agradecimientos: Esta investigación ha sido parcialmente financiada a través del proyecto PSI2010-21983C02-01 del Ministerio de Ciencia e Innovación y el Grupo de Investigación de la Junta de Andalucía HUM-820 (Lectura y Escritura en Español). 


\section{1.- INTRODUCCIÓN}

La importancia que el inglés, como lengua extranjera, ha ido adquiriendo dentro del panorama educativo español se pone de manifiesto desde la legislación educativa en el año 1970, con un aumento de la carga lectiva para esta materia en la enseñanza obligatoria. A partir de ese momento, las sucesivas leyes educativas han reflejado un incremento de la dedicación a la enseñanza de dicha materia. Actualmente, en la Ley Orgánica para la Mejora de la Calidad Educativa (L.O.M.C.E.), se reconoce en el artículo 18 la primera lengua extranjera como asignatura troncal. Así pues, el horario lectivo mínimo correspondiente no debe de ser inferior al 50\% del total del horario determinado por cada Administración.

La enseñanza del inglés cobra especial importancia dentro de la inmersión lingüística que se está experimentando en los últimos años en España; en ese sentido, se están instaurando programas bilingües en multitud de centros de Educación Primaria de las diferentes Comunidades Autónomas. Como consecuencia de la introducción del bilingüismo, la lengua extranjera ha pasado de ser un contenido de aprendizaje a ser un instrumento de aprendizaje. Este hecho afecta especialmente a los escolares ${ }^{1}$ con algún tipo de dificultad en la adquisición de una segunda lengua, ya que su problema puede pasar de ser específico del área curricular de lengua extranjera a generalizarse a otras áreas, como las matemáticas o el conocimiento del medio.

Por ello, para el profesorado especialista en Lengua Extranjera, es fundamental conocer las características de los escolares que pueden presentar dificultades en el aprendizaje de una segunda lengua. En concreto, el conocimiento de si dichas dificultades son específicas del área curricular o son una manifestación más de otros problemas que presenta el alumnado es especialmente relevante para una correcta intervención. En ese sentido, el objetivo de este artículo es examinar si las dificultades en el aprendizaje de una segunda lengua son una consecuencia más de otros problemas de aprendizaje que manifiesta el alumnado o si, por el contrario, hay escolares que evidencian una dificultad específica en el aprendizaje de la segunda lengua (es decir, en el resto de las áreas presentan un rendimiento adecuado).

El aprendizaje de una segunda lengua es un proceso complejo y, por ello, existen una gran cantidad de factores que influyen en su adquisición. Estos se pueden agrupar en tres ámbitos principales: ambientales, relacionados con el docente e individuales.

Entre los factores ambientales que favorecen la adquisición de una segunda lengua destacan: el contexto de aprendizaje (como el uso de buenos materiales, amplios recursos y/o número reducido de alumnos en clase), el beneficio del uso de la práctica distribuida (Beltrán, 1993) o el grado de semejanza entre la lengua materna y la lengua a aprender, ya que una mayor similitud hace que exista una menor dificultad en la adquisición de una nueva lengua (Martín, 2000).

Con respecto a los factores que están relacionados con el docente, uno de los más importantes es si el docente es nativo o también es su segunda lengua. Según Martín (1999), el docente nativo puede ejercer una buena labor por su conocimiento completo e implícito del idioma extranjero, sin embargo, el docente no nativo tiene como experiencia propia el haber aprendido esta lengua, previniendo mejor las dificultades que encontrarán los alumnos y ofreciendo una mayor empatía (Lakatos y Ubach, 1996; Martín, 1999; Medgyes, 2001). Otros factores relacionados con el docente que favorecen el aprendizaje son la especialización, la formación permanente y la motivación por la docencia (Imbernón, 1994).

Los factores individuales que afectan al aprendizaje de una segunda lengua son múltiples. Por ejemplo, se ha evidenciado que la edad es uno de los constituyentes más determinantes. En ese sentido, se defiende el aprendizaje desde edades tempranas, ya que el cerebro presenta una plasticidad que favorece nuevos aprendizajes (Díez, 2010; Mayo y Lecumberri, 2003; Mercau, 2009; Roca y Manchón, 2006; Singleton y Lengyel, 1995). Para Bley-Vroman (1989), el periodo más fructífero para

\footnotetext{
${ }^{1}$ Siguiendo las recomendaciones de la “Guía del Lenguaje no Sexista de la Universidad de Granada”, en este artículo se han usado, en la medida de lo posible, sustantivos genéricos (escolar, docente). No obstante, en otras ocasiones se ha optado por el uso del masculino genérico en lugar de las formas dobles (maestro/maestra; niño/niña) para evitar sobrecargar la lectura.
} 
aprender una lengua es cuando el cerebro y el sistema cognitivo han alcanzado la capacidad de realizar operaciones formales.

El nivel de lenguaje oral en la lengua nativa es otro importante factor individual, ya que la presencia de problemas en la lengua materna se extrapolarán a la segunda lengua (Maldonado, 1994). Por ello, es evidente que los escolares que presentan trastornos relacionados en el lenguaje oral, como el Trastorno Específico del Lenguaje, tendrán una alta probabilidad de mostrar problemas para el aprendizaje de una segunda lengua (Paradis y Crago, 2000).

Otro factor individual que puede afectar a la adquisición de una segunda lengua es que el escolar manifieste Dificultades de Aprendizaje (DA). Según los criterios diagnósticos del DSM-V (APA, 2014), este término se refiere a un grupo heterogéneo de trastornos caracterizados por dificultades persistentes en el aprendizaje de las habilidades académicas (lectura, escritura, expresión escrita y matemáticas). Los síntomas de las DA deben persistir al menos durante seis meses, incluso cuando se han ofrecido intervenciones dirigidas específicamente a su mejora. Además, las habilidades académicas afectadas deben estar significativamente por debajo del nivel esperable según la edad de la persona, causando una interferencia en el rendimiento de su actividad académica y vida diaria. Las DA no son debidas a trastornos intelectuales, sensoriales o lingüísticos, tampoco a una instrucción inadecuada ni a un ambiente en desventaja sociocultural.

Los tipos de dificultades de aprendizaje más estudiadas son los referidos a la adquisición de la lectura (dislexia), la escritura (disgrafía) y el cálculo (discalculia) (p.e.: Cuetos, 1991; García y Sánchez, 1998; Jimeno, 2004; Mata 2000; Miranda, Vidal-Abarca y Soriano, 2000). En el caso de los escolares con dislexia, están evidenciadas las dificultades que presentan para aprender una segunda lengua (p.e.: Arries 1999; Vellutino, Fletcher, Snowling, y Scanlon, 2004). Concretamente, su perfil se caracteriza por una adecuada adquisición del segundo idioma a nivel oral pero presentan problemas en actividades de lectura y/o escritura.

Según argumentan diferentes autores, las dificultades que presentan algunos individuos para aprender una segunda lengua serían generalizadas y se deberían a diferentes combinaciones de causas (McCardle, Mele-McCarthy y Leos, 2005; Lyon, citado en DiFino y Lombardino, 2004). Estas causas estarían relacionadas con los factores ambientales, del docente e individuales, como los descritos anteriormente. Por ejemplo, los alumnos pueden tener problemas previos asociados al aprendizaje de su lengua materna; concretamente, Vellutino y Scanlon (citado en Sparks y Ganschow, 1993) señalan que las causas serían dificultades en la fonología y/o de la sintaxis de la lengua nativa. En el mismo sentido, Arries (1999) también apoya el procesamiento fonológico como una de las problemáticas más frecuentes y destaca, como se ha comentado anteriormente, que la dislexia también sería otra causa de dificultad. Por su parte, García y Tyler (2010) argumentan que otros de los factores relacionados con las DA de una segunda lengua serían los problemas asociados con la adquisición de nuevo vocabulario, problemas en el procesamiento auditorio y visual y/o con la codificación y organización de información nueva.

Otros trabajos señalan como causa de la dificultad, un diseño poco adecuado del currículo, la ratio elevada de alumnos/clase, una baja formación del instructor, la falta de materiales específicos o metas poco realistas (DiFino y Lombardino, 2004). También la baja capacidad de memoria, falta de interés y/o de atención (Arries, 1999) o una incorrecta instrucción en su lengua materna (Maldonado, 1994). Otras variables importantes que se han puesto de manifiesto son las afectivas, como la falta de motivación, ansiedad y/o escasas estrategias de aprendizaje (Sparks y Ganschow, 1993).

No obstante, otros autores defienden la existencia de dificultades específicas en el aprendizaje de una segunda lengua. En este sentido, autores como García Mata (2001), consideran la DA de una segunda lengua como un problema específico de tipo cognitivo, más que por un problema general de toda actividad lingüística. Para Flax (citado en McCardle, Mele-McCarthy, y Leos, 2005), las dificultades en lengua extranjera podrían ser específicas aunque en muchas ocasiones son compensadas con motivación y una buena estimulación. Por último, Dulay (citado en Martín, 2000) argumenta que existen errores concretos de la segunda lengua (entre un 5\% y un 25\% los errores cometidos) que podrían atribuirse a una DA específica. 
Ante esta falta de consenso parece necesario aportar evidencia sobre la posible existencia de DA en una segunda lengua, con el objetivo de facilitar, al docente especialista, la identificación e intervención temprana de los escolares que puedan presentar estos problemas. Sin embargo, la revisión realizada de la literatura sobre esta temática no señala ningún estudio previo que aborde la posible existencia de una DA en el aprendizaje de una segunda lengua en población española. Por ello, el presente artículo tiene como objetivo general examinar si el fracaso en el área curricular de lengua extranjera en Educación Primaria puede ser explicable por otros problemas que manifieste el escolar o si, por el contrario, existe un tipo de dificultad específica de aprendizaje asociada a la adquisición de una nueva lengua.

Para ese objetivo, se han elaborado dos entrevistas semiestructuradas; una dirigida al docente especialista en inglés, y otra al tutor-maestro generalista. A través de la primera entrevista se pretende identificar a los niños que no superan los contenidos curriculares del área lengua extranjera. El propósito de la segunda entrevista es que el tutor facilite información de los niños anteriormente identificados, para detectar si presentan DA generalizadas (es decir, también obtienen bajas calificaciones en las demás áreas) o específicas (solo muestran bajas puntuaciones en el área de inglés). Una vez identificados los diferentes subgrupos, se pretende, como siguiente paso, determinar las posibles causas del bajo rendimiento en el aprendizaje de la segunda lengua.

\section{2.- MÉTODO}

\section{Participantes}

Los participantes de este estudio han sido 19 docentes: tres especialistas en Lengua Extranjera (inglés) y 16 tutores generalistas de tercero a sexto de Educación Primaria (EP). Los 19 docentes pertenecen a dos centros públicos con dos líneas cada uno, lo que equivale a un alumnado total de 384 escolares (cada clase incluía entre 23 y 25 alumnos).

Los dos centros escolares participantes se sitúan en una localidad de casi doce mil habitantes del área metropolitana de Granada. En general, el alumnado cuenta con un nivel socioeconómico medio.

El profesorado participante del centro 1 ha sido una maestra especialista que se ocupa de los cursos comprendidos entre tercero y sexto (unos 200 alumnos). Un total de veinticinco años de experiencia avalan a la docente, nueve de los cuales en el centro actual. Además, han participado ocho maestros generalistas, seis mujeres y dos hombres, que tutorizan los cursos de tercero a sexto.

Con respecto al centro escolar 2, han participado dos docentes especialistas (una maestra y un maestro), de los cuales uno de ellos se encarga de los dos grupos de tercero de EP y el otro docente del resto de los grupos de cuarto a sexto. Respectivamente, cuentan con seis y veintiún años de experiencia en el ámbito de la educación. Asimismo, ha colaborado ochos tutores generalistas (cuatro maestros y cuatro maestras) que participan en las mismas circunstancias descritas anteriormente para el centro 1.

\section{Instrumentos}

Los instrumentos utilizados en este trabajo han sido dos entrevistas semiestructuradas elaboradas siguiendo los objetivos propuestos. Una primera entrevista estaba dirigida para el maestro especialista de inglés con la finalidad de detectar a los escolares que presentaban unas bajas calificaciones en esta área curricular así como de recabar la opinión del profesorado acerca de las posibles causas de ese bajo rendimiento. La segunda, planteada como continuación de la primera, estaba destinada al maestro tutor de los niños identificados en la primera entrevista con bajo rendimiento en inglés, con el propósito de averiguar sus calificaciones en el resto de las áreas.

1.- Entrevista para el maestro especialista en Lengua Extranjera. Pretende detectar al grupo de alumnos que, por distintos motivos, no han adquirido los conocimientos mínimos y que, por lo tanto, no superan el área de inglés. Además, se indaga sobre las posibles causas de ese bajo rendimiento (desde la perspectiva del maestro especialista). 
La entrevista está compuesta por nueve cuestiones, mediante las cuales se busca obtener la siguiente información: nota media del alumnado que compone la clase para el área de inglés, alumnos que muestran menos participación e interés, olvidan realizar las tareas con frecuencia, obtienen puntuaciones más bajas y/o les cuesta mayor esfuerzo seguir el ritmo de la clase, razones que puedan explicar por qué estos alumnos no alcanzan el nivel medio, la opinión del docente sobre la posible existencia de dificultades específicas en el aprendizaje del inglés y sobre la influencia en el rendimiento de la asistencia a clases de inglés externas al colegio.

2.- Entrevista para el maestro tutor-generalista. Profundiza sobre el alumnado identificado en la entrevista anterior para comprobar su rendimiento en el resto de materias curriculares. En este caso, componen la entrevista diez preguntas focalizadas en los escolares que han sido identificados por el docente especialista. Estas cuestiones tratan sobre: la nota media de los alumnos identificados con bajo rendimiento en inglés en el resto de áreas curriculares, la existencia de algún trastorno diagnosticado, el desarrollo del lenguaje oral, ambiente socioeconómico y educativo en casa, conocimiento sobre si reciben algún tipo de apoyo en el colegio, el nivel de interés y participación en las áreas de conocimiento, la asistencia a clases complementarias en casa/academia, la realización habitual de los deberes y tareas en casa, el nivel de integración en su grupo de iguales y el reconocimiento de algún tipo de dificultad en el proceso de enseñanza y aprendizaje.

\section{Procedimiento}

En primer lugar, se pidió colaboración con la investigación a los directores de los centros, explicando la finalidad del proyecto. Una vez aceptada la participación por el equipo directivo, el procedimiento se dividió en dos fases estrechamente relacionadas, una primera centrada en los maestros especialista de inglés y otra segunda en el maestro generalista.

FASE 1: Entrevistas a los maestros especialistas en lengua extranjera (inglés). En el primer centro, se aplicaron un total de ocho entrevistas a la maestra especialista (una entrevista por cada curso donde imparte clase). La duración media de cada entrevista fue de seis-siete minutos y fueron completadas a lo largo de cuatro días.

A continuación, se realizaron las entrevistas en el segundo centro. En este caso, son dos los docentes que se reparten la carga lectiva del segundo y tercer ciclo de Educación Primaria para el área de inglés: uno de ellos completó dos entrevistas (correspondientes a los dos grupos de tercero) y el otro docente completó seis entrevistas (sobre los grupos de cuarto, quinto y sexto). La duración media fue también entre seis y siete minutos y fueron completadas en el tiempo del recreo a lo largo de una semana.

FASE 2: Entrevistas a los maestros tutores generalistas. Una vez identificados los niños con bajo rendimiento en el área de inglés gracias a las entrevistas realizadas a los especialistas en la fase 1, se continuó realizando las entrevistas a los maestros generalistas centradas en los niños identificados. En esta segunda fase, se realizaron entrevistas a 16 docentes cuya duración fue entre siete y ocho minutos por cada uno. El tiempo necesario para completar todas las entrevistas fue de 20 días. Tanto los especialistas como los tutores se mostraron colaborativos en todo momento y facilitaron la información requerida.

\section{3.- RESULTADOS}

Los resultados se presentan siguiendo las dos fases comentadas anteriormente en el procedimiento, referidas primero al análisis de las respuestas de los especialistas y, a continuación, a las de los maestros generalistas.

FASE 1: Entrevistas a los maestros especialistas en lengua extranjera (inglés). La Tabla 1 muestra el número total de escolares en cada curso (sumando las dos aulas por curso en los dos centros) y el número de alumnos detectados con bajo rendimiento en el área de inglés respecto a su grupo.

Se observa que el porcentaje de alumnos con bajo rendimiento en los cuatro cursos de tercero corresponde al 12,2\%, para cuarto curso es del 19,1\%, para quinto se encontró un 17,9\% y, finalmente, 
se ha detectado un 15,5\% para el último curso de EP. Así, se puede decir que $4^{\circ}$ de EP es el curso con un mayor porcentaje de estudiantes que no superan con éxito el área de inglés. Uniendo todos los estudiantes de segundo y tercer ciclo se obtiene un total del 16,1\% de escolares con bajo rendimiento en el aprendizaje del inglés.

Tabla 1.- Distribución del alumnado total y con bajo rendimiento en inglés por curso.

\begin{tabular}{lccccc}
\hline & $\mathbf{3}^{\mathbf{0}} \mathbf{E P}$ & $\mathbf{4}^{\mathbf{0}} \mathbf{E P}$ & $\mathbf{5}^{\mathbf{0}} \mathbf{E P}$ & $\mathbf{6}^{\mathbf{0}} \mathbf{E P}$ & TOTAL \\
\hline Total de alumnos escolarizados & 98 & 94 & 95 & 97 & $\mathbf{3 8 4}$ \\
Alumnos con bajo rendimiento en inglés & 12 & 18 & 17 & 15 & $\mathbf{6 2}$ \\
\hline
\end{tabular}

La entrevista también permitió obtener información acerca de los estudiantes con bajo rendimiento que recibían apoyo específico para el aprendizaje del inglés. Como se puede observar en la Tabla 2, un total de 32 niños asisten a un apoyo de una hora semanal que es impartido en los mismos centros por otro docente especialista en Lengua Extranjera. La selección de los escolares que recibían este apoyo, individualizado y personal, dependía del criterio del docente especialista.

Además, la Tabla 2 incluye el número de niños que también recibe apoyo externo al centro, como por ejemplo, a través de clases particulares o de asistencia a academias. En ese sentido, de los 62 niños con bajo rendimiento, solo 11 reciben además apoyo externo.

Tabla 2. Alumnado detectado con bajo rendimiento que reciben apoyo específico de la enseñanza del inglés.

\begin{tabular}{|c|c|c|c|c|c|}
\hline & $3^{\circ} \mathbf{E P}$ & $4^{\circ} \mathrm{EP}$ & $5^{\circ} \mathrm{EP}$ & $6^{\circ} \mathrm{EP}$ & TOTAL \\
\hline Alumnado detectado con bajo rendimiento en inglés & 12 & 18 & 17 & 15 & 62 \\
\hline Alumnado con apoyo de inglés solo en el centro escolar & 5 & 10 & 8 & 9 & 32 \\
\hline $\begin{array}{l}\text { Alumnado con apoyo de inglés en el centro escolar y fuera del } \\
\text { centro (clases particulares o academias) }\end{array}$ & 2 & 4 & 2 & 3 & 11 \\
\hline
\end{tabular}

Al mismo tiempo, se ha realizado un análisis respecto a los factores que podrían estar influyendo en la obtención de bajas calificaciones en inglés. El listado que aparece a continuación incluye algunas de las causas más comentadas por los docentes en la entrevista, ordenadas de mayor a menor frecuencia:

- Falta de motivación; alumnos desinteresados en la asignatura y/o con baja atención en clase.

- Poca responsabilidad y esfuerzo para realizar las tareas.

- Alumnos que están diagnosticados con Necesidades Educativas Especiales. En concreto, aquellos que reciben ayuda del pedagogo, del psicólogo del centro, del especialista de Audición y Lenguaje o Pedagogía Terapéutica.

- Problemas en el ambiente familiar, niños con desventaja sociocultural.

- Extrema inmadurez; niños que son más infantiles que sus compañeros.

Otra opinión generalizada entre los docentes especialistas entrevistados es la existencia previa de dificultades en el área de lengua. Así pues, se produce una extrapolación desde las dificultades que presentan en la lengua materna y ejercen una fuerte influencia en el aprendizaje de una nueva lengua.

Por último, en lo que se refiere a la cuestión de una posible existencia de dificultades específicas en el aprendizaje para una segunda lengua, los docentes especialistas siempre contestan de manera afirmativa. Además informan que, a lo largo de su carrera, han observado a niños con diferencias dentro de las cuatro destrezas de la competencia comunicativa (escuchar, hablar, leer y escribir), es decir, hay algunos niños que encuentran mayores problemas cuando el ejercicio que 
realizan está enfocado en la comprensión oral mientras que otros cuando se trata de la expresión verbal. De igual modo ocurre con la lectura y la escritura. En otros casos, hay niños que presentan una adquisición inadecuada de las cuatro habilidades.

FASE 2: Entrevistas a los maestros tutores generalistas. El análisis de las respuestas de la entrevista de los tutores tiene como objetivo discriminar entre los niños que presentan dificultades generalizadas (su rendimiento también es bajo en otras materias) y aquellos que presentan dificultades específicas en el aprendizaje de una segunda lengua (los tutores informan que presentan un rendimiento medio en el resto de las materias).

Como se puede observar en la Tabla 3, aunque la mayor parte de los niños detectados en la Fase 1 muestran dificultades en todas las materias, es decir, se considerarían con dificultades generalizadas, existe un porcentaje en cada grupo con dificultades específicas. Concretamente, dentro del total de la muestra de niños con bajo rendimiento, se ha encontrado un $21 \%$ de escolares con dificultades específicas de aprendizaje en la lengua extranjera. El análisis descriptivo por curso indica que en $3^{\circ} \mathrm{EP}$ y en $4^{\circ} \mathrm{EP}$ hay un $16,7 \%$ del total de niños detectados en la fase 1 , en $5^{\circ}$ se observa un $29,4 \%$ y en $6^{\circ}$ un $20 \%$.

Tabla 3. Distribución del alumnado con dificultades generalizadas y específicas en cada curso.

\begin{tabular}{|c|c|c|c|c|c|c|}
\hline & & $3^{\circ} \mathbf{E P}$ & $4^{\circ} \mathrm{EP}$ & $5^{\circ} \mathrm{EP}$ & $6^{\circ} \mathrm{EP}$ & TOTAL \\
\hline 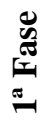 & $\begin{array}{l}\text { Alumnado identificado con bajo rendimiento por el } \\
\text { especialista en inglés }\end{array}$ & 12 & 18 & 17 & 15 & 62 \\
\hline \multirow{2}{*}{ 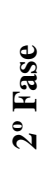 } & Alumnado con DA generalizadas & 10 & 15 & 12 & 12 & 49 \\
\hline & Alumnado con DA específicas del inglés & 2 & 3 & 5 & 3 & 13 \\
\hline
\end{tabular}

No obstante, sigue habiendo un número superior de DA generalizadas frente a las DA específicas. Es decir, el 79\% de los alumnos con bajo rendimiento en inglés también tienen problemas en las demás áreas por diversas razones (p.e.: problemas en lenguaje oral, necesidades educativas especiales...). También se desprende de las entrevistas que los niños con DA específicas en inglés suelen obtener, en las demás áreas, calificaciones que rondan el 6 (sobre 10) y no suelen destacar en ninguna materia escolar.

\section{4.- DISCUSIÓN Y CONCLUSIONES}

Este trabajo ha tenido como propósito examinar la existencia de posibles dificultades específicas en el aprendizaje del inglés. Para ello, se realizaron entrevistas a docentes en dos fases: una primera donde los especialistas de inglés identificaron alumnos con un bajo rendimiento en inglés y una segunda fase donde los maestros generalistas informaban del rendimiento de esos alumnos en el resto de materias. El objetivo fue averiguar si existía una discrepancia en el perfil de rendimiento de algunos alumnos, es decir, no superaban el área curricular de inglés pero sí el resto de las áreas. Asimismo, se pretendían examinar las causas más frecuentes de los problemas en el aprendizaje del inglés.

Según la información facilitada por los docentes especialistas en la primera fase, se ha identificado un total de 16,1\% como alumnos que no supera satisfactoriamente el área de inglés. Este resultado es relevante ya que es un porcentaje muy elevado de estudiantes que no consiguen alcanzar los objetivos de esta importante área curricular. Dentro de ese grupo de escolares que no superan el inglés, los docentes informan que el $21 \%$ presentaría una DA específica de una segunda lengua (inglés), es decir, un bajo rendimiento solo en esta materia. Incluso los docentes especialistas 
comentan que, a lo largo de sus años de experiencia, han observado discrepancias en lo que se refiere a las destrezas de habla, escucha, lectura y escritura. Estos resultados apoyan las propuestas de autores como García Mata (2001) o Martín (2000). No obstante, también es importante resaltar que estos estudiantes, a pesar de superar las diferentes materias, no destacaban en ningún área.

Siguiendo con el análisis pormenorizado de la muestra con bajo rendimiento, el profesorado indica que el $79 \%$ de estos escolares presenta dificultades generalizadas, por tanto, no existe una discrepancia ya que su rendimiento es bajo en todas las materias. Este resultado iría en la línea de trabajos como el realizado por Ganschow, Sparks, Javorsky, Pohlman, y Bishop-Marbury (1991) o Maldonado (1994). Las causas a las que los docentes atribuyen este fracaso son una combinación de factores ambientales y/o individuales, así como la interacción entre ellos. En ninguna entrevista se ha hecho referencia a factores relacionados con el docente que puedan explicar, al menos en parte, algunas de las dificultades observadas (por ejemplo, la necesidad de una mayor formación).

La existencia de DA específicas y generalizadas es un conocimiento relevante para el profesorado especialista en inglés, ya que es posible que el enfoque de intervención sea diferente para cada tipo de problemática. Este hecho ya ocurre con otras DA, como la dislexia, donde el perfil de dificultades de cada individuo determina la orientación de la intervención (Jiménez-Fernández, Defior y Serrano, 2012). Por ello, el docente especialista debe ser consciente de la posibilidad de encontrar alumnos con diferentes perfiles de dificultad en el aprendizaje de una lengua extranjera (específica o generalizada) y de la necesidad, por tanto, de desarrollar una intervención individualizada y personalizada en cada caso. Asimismo, esta labor debería ser sustentada por los organismos educativos; al igual que ocurrió en su momento con materias tales como Lengua o Matemáticas, se va haciendo imprescindible la creación de un apoyo específico en Lengua Extranjera para que los centros puedan dar respuesta a las necesidades específicas del alumnado. En este sentido, los dos centros participantes en esta investigación persiguen esta idea, de modo que están llevando a la práctica un programa de apoyo específico en la materia de inglés.

No obstante, esta investigación presenta una limitación relacionada con el reducido tamaño de la muestra ya que solo han participado dos centros escolares. Este hecho limita la generalización de los resultados obtenidos, aunque es importante resaltar que la participación de los dos centros ha permitido la obtención de información de aproximadamente unos 400 alumnos de segundo y tercer ciclo.

Por otra parte, se ha utilizado como único instrumento la entrevista diseñada para esta investigación, por lo que se ha obtenido solamente la perspectiva del profesorado. A pesar de la gran precisión que presentan juicios de los maestros (Hoge y Coladarci, 1989), hubiera sido muy enriquecedor aplicar pruebas estandarizadas a los estudiantes seleccionados para indagar posibles factores causales de su bajo rendimiento y así explicar la dificultad específica en inglés.

Partiendo de esta situación, como continuación de este primer estudio exploratorio, se plantea la posibilidad de realizar futuros trabajos que incrementen el número de centros participantes para que los resultados fueran más representativos. Igualmente, se podrían incluir evaluaciones psicopedagógicas a los niños que han sido detectados con DA específicas en el inglés, así como entrevistas a las familias. También próximas investigaciones podrían estar focalizadas en discriminar los métodos más efectivos de enseñanza del inglés tanto para niños con DA específicas como generalizadas.

En conclusión, este trabajo aporta datos novedosos sobre la existencia de una DA específica para aprender una segunda lengua. Es solo un punto de partida para seguir confirmando e indagando esta DA con la finalidad última de conseguir desarrollar estrategias de intervención individualizadas que permitan a los escolares adquirir y desenvolverse adecuadamente en el dominio de una segunda lengua.

\section{5.- REFERENCIAS}

American Psychiatric Association, APA (2014). Guía de consulta de los criterios diagnósticos del DSM-V. Madrid: Panamericana. 
Arries, J. F. (1999). Learning disabilities and foreign languages: A curriculum approach to the design of inclusive courses. The Modern Language Journal, 83(1), 98-110.

Beltrán, J. (1993). Procesos, estrategias y técnicas de aprendizaje. Madrid: Síntesis.

Bley-Vroman, R. (1989). What is the logical problem of foreign language learning. Linguistic Perspectives on Second Language Acquisition, 4, 1-68.

Cuetos, F. (1991). Psicología de la escritura: diagnóstico y tratamiento de los trastornos de la escritura. Madrid: Escuela Española.

Díez, V. A. (2010). El inglés mejor a edades tempranas. Pedagogía Magna, 5, 251-256.

DiFino, S. M. y Lombardino, L. J. (2004). Language learning disabilities: the ultimate foreign language challenge. Foreign Language Annals, 37(3), 390 - 400.

Ganschow, L., Sparks, R. L., Javorsky, J., Pohlman, J., y Bishop-Marbury, A. (1991). Identifying native language difficulties among foreign language learners in college: A "foreign" language learning disability? Journal of Learning Disabilities, 24(9), 530-541.

García Mata, J. (2001). Agrupamiento, estilo de aprendizaje y trabajo basado en proyectos en la formación de maestros no especialistas en lengua extranjera. Málaga: Universidad de Málaga.

García, J. N. y Sánchez, J. N. G. (1998). Manual de dificultades de aprendizaje: lenguaje, lecto-escritura y matemáticas. Madrid: Narcea Ediciones.

García, S. B. y Tyler, B. J. (2010). Meeting the needs of English language learners with Learning Disabilities in the General Curriculum. Theory Into Practice, 49(2), 113-120.

Hoge, R. D. y Coladarci, T. (1989). Teacher-based judgments of academic achievement: A review of literature. Review of Educational Research, 59(3), 297-313.

Imbernón, F. (1994). La formación y el desarrollo profesional del profesorado: hacia una nueva cultura profesional. Barcelona: Graó.

Jiménez-Fernández, G., Defior, S., y Serrano, F. (2012). Perfiles de dificultad en la dislexia evolutiva: lectura imprecisa vs. lectura no fluida. En AA.VV., Actas del XXVIII Congreso Internacional de AELFA. Sección Casos Clínicos (pp. 538-545). Madrid: UCM.

Jimeno, P. (2004). La enseñanza de la expresión escrita en todas las áreas. Gobierno de Navarra, Departamento de Educación: Blitz serie roja.

Lakatos, S. y Ubach, A. (1996). ¿Profesor nativo o no nativo?. En Actas del VI Congreso Internacional de ASELE. Tendencias Actuales en la Enseñanza del Español como Lengua Extranjera II (pp. 239-244). León: Universidad de León.

Ley orgánica para la mejora de la calidad educativa (LOMCE) (Ley Orgánica 8/2013, 9 de diciembre). Boletín Oficial del Estado, $n^{\circ}$ 295, 2013, 10 diciembre.

Maldonado, J. A. (1994). Bilingual special education: Specific learning disabilities in language and reading. Journal of Education Issues of Language Minority Students, 14(2), 127- 147.

Martín, J. M. (1999). El profesor nativo de español. En Actas del X Congreso Internacional de la ASELE. ¿Qué español enseñar? Norma y variación lingüísticas en la enseñanza del español a extranjeros (pp. 433438). Zaragoza: Universidad de Zaragoza.

Martín, J. M. (2000). La lengua materna en el aprendizaje de una segunda lengua. Sevilla: Universidad de Sevilla.

Mata, F. S. (2000). Cómo prevenir las dificultades en la expresión escrita. Málaga: Aljibe.

Mayo, M. D. P. G. y Lecumberri, M. L. G. (2003). Age and the acquisition of English as a foreign language. Multilingual Matters: Clevedon.

McCardle, P., Mele-McCarthy, J., y Leos, K. (2005). English language learners and learning disabilities: research agenda and implications for practice. Learning Disabilities Research y Practice, 20(1), 68-78.

Medgyes, P. (2001). When the teacher is a non-native speaker. Teaching English as a second or foreign language, 3, 429-442.

Mercau, M. (2009). La enseñanza escolar temprana en inglés. Casa del Tiempo, 4(24), 43-46.

Miranda, A., Vidal-Abarca, E. y Soriano, M. (2000). Evaluación e intervención psicoeducativa en dificultades de aprendizaje. Madrid: Pirámide. 
Paradis, J. y Crago. M. (2000). Tense and temporality: A comparison between children learning a second language and children with SLI. Journal of Speech, Language, and Hearing Research, 43(4) 834-842.

Roca, J. y Manchón, R. (2006). Algunas consideraciones sobre el factor edad en relación con la enseñanza de las lenguas extranjeras en la escuela. Porta Linguarum, 5, 63-76.

Singleton, D. M. y Lengyel, Z. (1995). The age factor in second language acquisition: A critical look at the critical period hypothesis. Multilingual Matters.

Sparks, R. y Ganschow, L. (1993). Searching for the cognitive locus of foreign language learning difficulties: linking first and second language learning. The Modern Language Journal, 77(3), 289-302.

Vellutino, F. R., Fletcher, J. M., Snowling, M. J., y Scanlon, D. M. (2004). Specific reading disability (dyslexia): What have we learned in the past four decades? Journal of Child Psychology y Psychiatry, 45(1), 2-40.

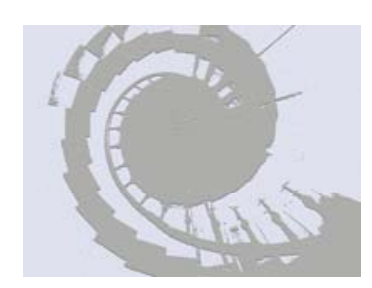

